\title{
Internet-Based Hearing Assessment During COVID era in Indian Population: Practical and Safe Option
}

\author{
Pranav Mishra $^{1} \cdot$ Angira Katyayan $^{2} \cdot$ Adri Katyayan $^{3} \cdot$ Anupam Mishra $^{1}$ (I)
}

Received: 19 August 2020/Accepted: 3 October 2020/Published online: 20 October 2020

(C) Association of Otolaryngologists of India 2020

\begin{abstract}
This pilot observation intends to stress on webbased hearing assessment (WBHA) as somewhat parallel to clinical pure tone audiometry. While WBHA was comparable with PTA in context of severity of deafness particularly in symmetrical hearing loss, it was inconclusive for a gross asymmetry despite multiple trials. With increasing COVID transmission, more need for social distancing and lack of audiologists in developing countries, the self-participation by patients in WBHA model will prove to be a very safe model of deafness-screening.
\end{abstract}

Keywords Audiometry $\cdot$ Internet $\cdot$ Deafness

The social proximity particularly in a closed cabin such as audiological suit portends a risk for COVID disease transmission. Hence in accordance with social distancing, tele-assessment of hearing functions are to be preferred wherever necessary. This context is particularly applicable for rural sectors of developing world with a higher prevalence of hearing loss. A WHO study ${ }^{1}$ conducted by our team, revealed a prevalence of $15 \%$ deafness in general rural population across Lucknow district (India) that is in turn grossly mismanaged owing to a substandard health care $^{2}$ and patient-doctor ratio. COVID-19 has altered the

Anupam Mishra

amishra_ent@yahoo.com

1 King George Medical University, Lucknow, Uttar Pradesh 226003, India

2 Indian Institute of Technology (IIT), Kanpur, Uttar Pradesh 208016, India

3 City Montessori School, Mahanagar, Lucknow, Uttar Pradesh 226006, India existing paradigm in hearing assessment techniques and will further worsen the deafness in densely populated third world. Hence there is a dire need to at least screen out the potential hearing impaired in general population with absolutely safe and reliable methods. This commentary highlights a pilot study intended for screening hearing using internet that can be safely applied to this COVID situation.

Considering the least expensive internet services available in India (across the globe), this pilot work supports 'internet-based hearing assessment model' to screen the targeted population even by non-medical persons and thereby also overcoming geographical barriers. Several such online programs are freely available particularly linked with sale of hearing aids. Through observer ship program of undergraduate and high school students in a tertiary hospital (after due permission from Chief Medical Superintendent of Hospital), a basic comparison of subjective (air conduction) responses from pure tone audiometry (PTA) and online web-based hearing assessment (WBHA) was undertaken. The latter consisted of a simple earphone connected to a laptop with Internet that generated similar pure tones across 6 speech frequencies as PTA. Twenty patients of hearing loss were assessed both by PTA and WBHA. Two patterns of deafness were identified by PTA (1) symmetrical (possibly resulting from prebyacusis, chronic noise exposure, ototoxic drugs etc.) and (2) asymmetrical (ear discharge, trauma etc.). WBHA was comparable with PTA in context of severity of deafness particularly in symmetrical hearing loss, but was inconclusive for a gross asymmetry despite multiple trials. Moreover time of assessment in WBHA was somewhat more than PTA but still comparable in minimal symmetrical hearing loss category. The speed of internet server was instrumental in this variation. It is also noteworthy that 
PTA was conducted by qualified audiometricians while subjective responses in WBHA were noted by non-medical untrained students (under the direct supervision of consultant). The students had however tried WBHA on themselves for sensitizing themselves before observing in clinic.

WBHA can at least help today's world in screening deafness resulting from presbyacusis, chronic noise exposure and some categories of ototoxicity. Furthermore for computer illiterate rural population, a computer student (of primary school) residing at home may facilitate WBHA. In addition regular home self-checkups at for any potential improvement in deafness can also be monitored. Even a failure of identifying asymmetrical deafness (unilateral otitis media), this 'WBHA-inconclusive-status' may further help in referring to otologic center. Hence such a hearing-screening-program can be universally adopted to facilitate social distancing and selecting the target population that needs a formal audiological assessment in closed sound proof cabins with ideal environment. In addition the establishment of unmanned 'Hearing-Kiosks' at suitable places may further facilitate self-screening wherein using personal earphones will minimise corona spread. Not the least future of WBHA lies in linking it with purchasing online hearing aid as well, thereby reducing the incurred cost.

It is to be emphasized that WBHA is not a substitute of PTA that in turn has many more functions such as bone conduction, speech testing etc. WBHA may be a good screening tool for candidates that need to undergo PTA. With a highly infectious scenario in the background of COVID and considering the scarcity of otologists/ audiologists in developing countries, the actual patient-participation in such WBHA models will definitely prove out to be a very safe model of limiting community deafness.

- This write up is Compliant with Ethical Standards and there are no potential conflicts of interest

- The routine hearing assessment was carried out in the outpatient clinic as usual after properly explaining the patients. Even the computerised audiometry performed in routine OPD is carried out the same way. All the 20 patients were well informed about the audiological procedure and their verbal consent was duly obtained before their participation.

Acknowledgements The authors would primarily like to acknowledge ARTH-DIGITAL for technical support. In addition Dr SN Sankhwar CMS GMAH is acknowledged for giving due permission to carry out the testing under supervision. Lastly Prof Veerendra Verma, Dr Pankaj Pandey, Dr Akshay Mishra and audiologists viz. Mr Varun, Mr Chandan, Mrs Amita Srivastava are to be acknowledged for facilitating patient referral and audiology testing.

Author contributions Pranav Mishra conceptualized the study with ARTH-DIGITAL and identified the logistics of online assessment. As a Student in CA he did a couple of days observation at the Department of Otolaryngology at King George Medical University Lucknow.

Anupam Mishra planned the study and organised a team, obtained due permission for carrying out the study in the university hospital and thereafter the study was conducted under supervision of Anupam Mishra. The results were analysed and final report was approved by Anupam Mishra.

Angira Katyayan, Pranav Mishra and Adri Katyayan as non-medical investigators helped in registering patient's responses, collecting data and literature search. The report was prepared by all the three that was further edited by Anupam Mishra before final approval.

All the authors had their consensus regarding approval before submission.

Funding: No external or internal funding except only technical (with material in kind) support by ARTH-DIGITAL.

Compliance with ethical standards

Conflict of interest None.

\section{References:}

1. Mishra A, Verma V, Shukla GK, Mishra SC, Dwivedi RC (2011) Prevalence of deafness in indian population: an epidemiological study for health policy considerations. Indian J Public Health 55(2):132-134

2. Clark J (2015) Indian medical education system is broken Reuters investigation finds. BMJ 350:h3324. https://doi.org/10.1136/ bmj.h3324

Publisher's Note Springer Nature remains neutral with regard to jurisdictional claims in published maps and institutional affiliations. 